\title{
A Binational Model of Collaboration for Enhancing Cross-border ID Surveillance
}

\author{
Kristine Ortwine $^{\star 2,1}$, Karen Ferran ${ }^{1}$ and Esmeralda Iniguez-Stevens ${ }^{1}$ \\ 'Early Warning Infectious Disease Surveillance, California Department of Public Health, San Diego, CA, USA; ${ }^{2}$ San Diego State \\ Graduate School of Public Health, San Diego, CA, USA
}

\section{Objective}

The purpose of this demonstration is to describe the cross-border collaborative processes used for the development of a transparent methodology to identify and prioritize zoonotic infectious disease agents in the California-Baja California border region.

\section{Introduction}

International borders present unique challenges for the surveillance of infectious disease. Border communities represent locations with vast differences in cultures and languages, governing institutions, healthcare access, and priorities for the collection and surveillance of disease data. Pathogens and the health and security risks they create do not respect geographical and political boundaries. However, the organizations responsible for the surveillance and control of these agents must function within the borders of their respective governments. One Border One Health $(\mathrm{OBOH})$ is a binational, multidisciplinary initiative aimed at engaging partners in the US and Mexico to identify and implement methods for successful communication and collaboration to enhance health capacity and disease surveillance within the border region. The advancements of international initiatives such as $\mathrm{OBOH}$ will help to develop the types of multi-country networks necessary for the effective monitoring of disease patterns and risks.

\section{Methods}

One Border One Health Surveillance Committee participants represent multi-disciplinary professionals working together for the advancement of One Health principles in the California/Baja California border region. This showcase documents the identification and prioritization of zoonotic infectious disease agents along the US-Mexico border, by use of a transparent methodology which engaged public and private partners from both countries. Preliminary research and input from collaborators in government, academic, and private sectors in the US and Mexico allowed for review and discussion of current methodologies available for prioritizing infectious agents. The DISCONTOOLS Work Package 2 Prioritization Scoring Model was selected as the basis for scoring and weighting various zoonotic diseases of concern within border region. Subject matter experts were then asked to review and score an initial list of diseases, in order to produce a final ranked list of pathogens. The intent is that these prioritized pathogens will be used by government agencies to make informed decisions, integrating priorities from both nations with regards to infectious disease surveillance. This collaboration provides insight into the binational cooperation needed for the selection of diseases to be considered in a regional, integrated disease surveillance system. To the authors' knowledge this is the first transparent scientific-based approach to pathogen prioritization in the US-Mexico border region.

\section{Conclusions}

$\mathrm{OBOH}$ is the first binational regional network of its kind along the US-Mexico border recognizing the interconnectivity between human, animal, and environmental health. Given the limited resources in the current economic climate, the use of regional integrated surveillance systems provide an opportunity to protect and improve border health and security by moving away from species-specific surveillance programs. The process showcased here for the transparent review and prioritization of pathogens along the California-Baja California border can be used as a model along the entire US-Mexico Border. The ultimate aim is to protect border communities through the creation of a binational, early warning surveillance system which would allow for actionable and timely interventions to limit emergence, mitigate spread, provide gap analysis, and enhance prevention and control for several emergent and re-emergent diseases. Ultimately, this will decrease negative health and environmental impacts while improving agricultural and economic outcomes in both nations. However, obstacles such as continued sustainability, identification of new multidisciplinary collaborators, cooperation between government agencies, and identifying funding for advancement of integrated regional surveillance systems remain challenges.

\section{Keywords}

One Health; disease surveillance; cross-border collaborative

\section{*Kristine Ortwine}

E-mail: kristine.ortwine@cdph.ca.gov 